\title{
On the Production of Useful Knowledge
}

\author{
SANDRO GALEA
}

He United STATES IS MARKED By A PROFOUND MiSMatCH
between our avowed interest in health and our limited invest-
ment in the areas that actually generate population health. ${ }^{1}$ This has resulted in the United States spending far more on health care than any other country, while having worse health indicators than nearly all its peer high-income countries. ${ }^{2}$

The challenge then becomes, what can we do about this? Much of the responsibility lies with the political and public sector actors who have long had the greatest influence on improving population heath. However, some of the responsibility also lies with the academy. Policymakers may drift from what we call the best evidence, but a mature system of checks and balances aspires to hold policymakers accountable and one hopes that in the fullness of time policies and actions will drift toward evidence-based solutions-that is, when evidence is available.

This challenges the academy to generate useful data about the foundational drivers of health so that we can build policies on these data, nudging the health of populations forward. If we recognize that poor-quality housing, limited transportation, income gaps, and absence of adequate supports for marginalized populations (to name but a few) are all responsible, in part, for the generation of population health in the United States, then it stands to follow that academicians and scholars should have abundant data to back this up.

This is, unfortunately, not the case. A review of the best summary resource for data in this area-the Centers for Disease Control and Prevention (CDC) Health Impact in 5 Years initiative (https://www. cdc.gov/policy/hst/hi5/index.html)—illustrates the nature of the problem. Much of the data on the health impact and cost-effectiveness of areas such as public transportation system introduction or expansion, early childhood education, and the earned-income tax credit (EITC) are

The Milbank Quarterly, Vol. 95, No. 4, 2017 (pp. 722-725)

(C) 2017 Milbank Memorial Fund. Published by Wiley Periodicals Inc. 
a decade old and, in many respects, leave more questions unanswered than they do provide clear and compelling answers.

How many lives will be saved by expanding transportation networks? How many quality-of-life years will be gained by expanding the EITC? What is the cost-effectiveness of universal prekindergarten education? These questions are left wanting, and a systematic review of the literature does little to reassure us.

This prompts two questions.

\section{Why Are Data on the Foundational Drivers of Population Health So Lacking?}

First, studies about the relation between foundational drivers and population health are, simply, hard to conduct. The dominant approach to biomedical research has long been a deterministic one in which we use forms of linear models to isolate factors that we consider to be causes of particular health indicators. This has led to the development of methodological approaches that lend themselves more readily to the isolation of individual-level biological or perhaps behavioral causes than to grappling with larger-scale social, political, or cultural factors that are complex and not readily reduced to a simple single variable. The blurring of methodological approaches among social policy research, political science, sociology, and epidemiology is gradually introducing methods and approaches that can be useful in this regard, ${ }^{3}$ but progress is slow.

Second, data that can ground studies on the relation between foundational factors and health are hard to come by. Foundational forces are typically in the hands of large-system administrators, and this group has much less incentive to collect data systematically, or to make it available, than do researchers. There are some exceptions. For example, the Department of Housing and Urban Development's Moving to Opportunity Study, has yielded a trove of data that has advanced our understanding of the foundational influences on health. By and large, however, we tend to experience a disconnect between those who have the potential to generate data that can usefully inform population health science and those in the academy who are charged with conducting sound studies using such data. 


\section{What Can Be Done to Encourage the Generation of Knowledge Useful for the Production of Population Health?}

Two solutions present themselves that can nudge us forward on the generation of data useful to population health-both, perhaps not surprisingly, building on the challenges noted earlier.

First, it behooves us to invest in the intellectual hard work that can articulate and advance population health science. Such an effort can sharpen our thinking about what kind of studies we need, what data will advance our cause, and what methodological approaches can indeed inform the practice of forward-looking public health. Recent efforts to articulate the remit of population health science ${ }^{4}$ and the enthusiasm that has greeted the notion of Public Health 3.0 articulated by the US Department of Health and Human Services serve as signs for optimism. ${ }^{5}$ It will, of course, take time for these ideas to penetrate and to inform thinking in the scientific community, but these first steps are promising.

Second, we need to creatively catalyze collaboration between the sectors who generate data that can answer foundational questions with scholars that can generate the population health science we need. It may seem trite to ask for intersectoral collaboration between the academy and public health practice. I would suggest that we will only make progress in this regard if incentives are aligned within both the practice and the academic community to generate scholarship that can be useful to advancing population health. For example, a stepped-up effort within public health agencies to incentivize research would encourage programmatic alignment with research and reward the generation of data and its dissemination.

In short, improving the state of American health requires the collective recognition that we are misspending our resources. We need to shift from ever more spending on health care, especially the technologies, procedures, and treatments that have been proven to not make much difference in the lives of Americans, and instead direct those funds toward spending on the foundational drivers of population health. This must include the generation of knowledge that can better inform such an effort, and an embrace of responsibility toward that end by the academy. Identifying the barriers to such an effort and adopting modest solutions might be first steps in this 
direction. A more concerted collective effort to rectify what is truly a fundamental barrier to advancing population health science should follow.

\section{References}

1. Galea S. An unhealthy mismatch. Milbank Q. 2017;95(2):229-232.

2. Woolf S, Aron L, eds. US Health in International Perspective. Shorter lives, poorer health. Washington, DC: National Research Council and Institute of Medicine; 2012.

3. Marshall BDL, Galea S. Formalizing the role of complex systems methods in causal inference and epidemiology. Am J Epidemiol. 2015;181(2):92-99.

4. Keyes KM, Galea S. Setting the agenda for a new discipline: population health science. Am J Public Health. 2016;106(4):633-634.

5. DeSalvo KB, O'Carroll PW, Koo D, Auerbach JM, Monroe JA. Public health 3.0: time for an upgrade. Am J Public Health. 2016;106(4):621-622.

Address correspondence to: Sandro Galea, MD, DrPH, School of Public Health, Boston University, 715 Albany St, Talbot 301, Boston, MA 02118 (email: sgalea@bu.edu). 